\title{
Electronic Properties of Graphene Nanoribbons With Defects
}

\author{
Konstantinos Rallis (), Member, IEEE, Panagiotis Dimitrakis (), Senior Member, IEEE, Ioannis G. Karafyllidis (), \\ Antonio Rubio ${ }^{(1)}$, Senior Member, IEEE, and Georgios Ch. Sirakoulis ${ }^{\circledR}$, Member, IEEE
}

\begin{abstract}
Graphene nanoribbons (GNRs) are the most important emerging Graphene structures for nanoelectronic and sensor applications. GNRs with perfect lattices have been extensively studied, but fabricated GNRs contain lattice defects the effect of which on their electronic properties has not been studied extensively enough. In this paper, we apply the Non-Equilibrium Green's function (NEGF) method combined with tight-binding Hamiltonians to investigate the effect of lattice defects on the conductance of GNRs. We specifically study, butterfly shaped GNRs, which operate effectively as switches, and have been used in CMOS-like architectures. The cases of the most usual defects, namely the single and double vacancy have been analytically examined. The effect of these vacancies was computed by placing them in different regions and with various numbers on GNR nano-devices, namely edges, main body, contacts and narrow regions. The computation results are presented in the form of energy dispersion diagrams as well as diagrams of maximum conductance as a function of the number of lattice defects. We also present results on the defect tolerance of the butterfly shaped GNR devices.
\end{abstract}

Index Terms-Defects, graphene, graphene nanoribbons (GNRs), nanoelectronics, nanoscale devices, non-equilibrium green 's function (NEGF).

\section{INTRODUCTION}

$\mathbf{G}$ RAPHENE, is a two-dimensional (2D) material that was effectively isolated in 2004 and exhibits unique mechanical, electrical, optical and thermal properties [1], [2]. At the simplest and most interesting configuration, graphene is a single layer of carbon atoms that are organized as a honeycomb lattice. Single Layer Graphene (SLG) is formed at large areas by CVD method on $\mathrm{Cu}$ foils. The fabrication method makes unavoidable

Manuscript received November 16, 2020; accepted January 18, 2021. Date of publication January 27, 2021; date of current version February 26, 2021. The work was supported by Hellenic Foundation for Research and Innovation (HFRI) under the HFRI Ph.D. Fellowship Grant (Fellowship no. 1243). The review of this article was arranged by the guest editors for NANOARCH2020. (Corresponding author: Konstantinos Rallis.)

Konstantinos Rallis is with the Department of Electrical and Computer Engineering, Democritus University of Thrace, Xanthi 67100, Greece, and also with the Department of Electronics Engineering, Universitat Politécnica de Catalunya, Barcelona 08034, Spain (e-mail: krallis@ee.duth.gr).

Ioannis G. Karafyllidis and Georgios Ch. Sirakoulis are with the Department of Electrical and Computer Engineering, Democritus University of Thrace, Xanthi 67100, Greece (e-mail: ykar@ee.duth.gr; gsirak@ee.duth.gr).

Panagiotis Dimitrakis is with the Institute of Nanoscience and Nanotechnology, NCSR Demokritos, Athens 15310, Greece (e-mail: p.dimitrakis@inn.demokritos.gr).

Antonio Rubio is with the Department of Electronics Engineering, Universitat Politécnica de Catalunya, Barcelona 08034, Spain (e-mail: antonio.rubio@upc.edu).

Digital Object Identifier 10.1109/TNANO.2021.3055135 the presence of structural defects [3]. These are mainly attributed to the process conditions such as the growth rate [4], and the imperfections of the metallic foils [5]. In addition, the harsh process of the graphene during the transfer on the desired substrate and/or device fabrication induces lattice defects [6]. The SLG defects are distinguished to intrinsic and extrinsic [7], where the first term refers to structural imperfections of the honeycomb lattice, while the last one to defects due to non-carbon atoms or chemical groups attached on the SLG lattice. Experimentally, the SLG defects were investigated by Raman spectoscopy [8] and transmission electron microscopy (TEM) [9] regarding their structural properties and by Hall and conductivity measurements regarding their transport properties. Profound theoretical works and reviews [6], [10] have been published on the effect of lattice defects on the transport properties of graphene, where tight-binding [11], ab initio and combined methods have been examined [10]. Among the various predicted characteristics of a defective graphene lattice, which have been found experimentally and modeled successfully, is conductivity engineering of graphene sheets by controlling the structural defects [12]. Although it is well-known that lattice defects always reduce the mobility - and hence the conductivity of graphene due to scattering effects $-i$ thas been demonstrated that defects can also improve the conductivity through the generation of carriers. In the same direction, it has been proven that the metal/graphene contact resistance is remarkably decreased when extended defects are introduced in the graphene lattice underneath the metal electrodes [13], [14].

Obviously, defects are expected to affect severely the properties of graphene sheets as their size (mainly the width $W$ ) decreases and becomes the so called graphene nanoribbon (GNR), which is the most emerging structure for graphene low power electronic devices and sensors [15], [16]. The attractive properties of GNRs arise from quantum confinement and the edge form (zigzag or armchair) of the GNR lattice. The spatial confinement leads to momentum and consequently to discrete energy bands $(\Delta E \sim 2 \mathrm{eV} / W(\mathrm{~nm}))$ and conductance quantization, while the armchair GNR can be either metallic or semiconducting depending on the size $W$ of the GNR. It has been theoretically predicted and experimentally proven that when a GNR is below $10 \mathrm{~nm}$ shows semiconducting properties despite the metallic one in bulk [17]. Several theoretical studies on GNR defects have been published [18]-[21]. Recently, theoretical investigations have demonstrated that GNRs can be used to form $\mathrm{p}-\mathrm{n}$ junctions [22], and complementary logic gates [23] with superior 
delay time compared to CMOS [24], as well as Multi Value Logic circuits [25]. Furthermore, it has been predicted by simulation that depending on the shape of the GNR it is possible to create energy pseudo-gaps where conductivity is zero and thus to create a full set of logic gates by engineering the GNR shape [26], [27].

In this paper, we explore the effect of the most common lattice defects on the conductivity of a GNR combining Tight-Binding Hamiltonian (TBH) with the Non-Equilibrium Green's Function (NEGF) method and the Landauer formalism for the conductivity calculation, in terms of the defect location and concentration. The cases of the simplest possible defects, namely the single and double vacancy have been analytically examined. Those vacancies were tested in different regions and concentrations on the GNR nanodevice, like edges, main body, contacts and narrow region. The corresponding results are presented in the form of energy dispersion diagrams, maximum conductance to number of lattice defects diagrams, as well as change of energy gap to defect density diagrams, indicating the varying defect tolerance of the butterfly shaped GNR devices. With this study, we further investigate these lattice effects aiming to aid the design of real nanoelectronic circuits in which defects will always be present. We also provide insights in the previously unexplored field of defects on GQPC devices, which is crucial for their viability as devices of computational circuits. The aforementioned analysis, with the addition of defect locality as a parameter, comprise a tool for a rough estimation of defect density and locality in real devices, which is obtained by only measuring the conductance of the device. The rest of the manuscript is structured as follows. In Section II, we describe the modeling methodology and the theoretical aspects of the proposed simulation. In Section III to VII, the simulation results on the effect on the conductivity of the defects in various regions of the GNR are presented and thoroughly discussed. Finally, Section IX summarizes the results and draws the main conclusions.

\section{Modelling AND MethoD}

The calculation of conductance of a GNR was achieved by combining the Tight-Binding Hamiltonian (TBH) method [28], [29] and the Non-Equilibrium Green's Function (NEGF) method [30], [31], which is considered to be the state of the art modeling method for atomic level modeling of nanoscale devices, and, in particular, carrier transport, which are governed by quantum mechanical effects. The combination of the two aforementioned methods enables us to perform computations for any possible nanoribbon shape. In this work, we are considering the butterfly shape (quantum-point contact) [32], [33], that is a GNR comprising a graphene nanostripe, called hereby channel, ending at both sides to large trapezoid graphene regions, called hereby contacts, as shown in Fig. 1. In terms of dimensions, the device under investigation has a total length of $6.026 \mathrm{~nm}$ $(30 \sqrt{2} \alpha)$. The width on the short region is equal to $1.562 \mathrm{~nm}$ $(11 \alpha)$, while the maximum width of the large region is equal to $5.822 \mathrm{~nm}(41 \alpha)$, where $\alpha$ is the lattice constant equal to $0.142 \mathrm{~nm}$, but the results are similar also for higher dimension devices. The edges of the GNR were selected to have zigzag configuration. Our calculations were focused on the dependence
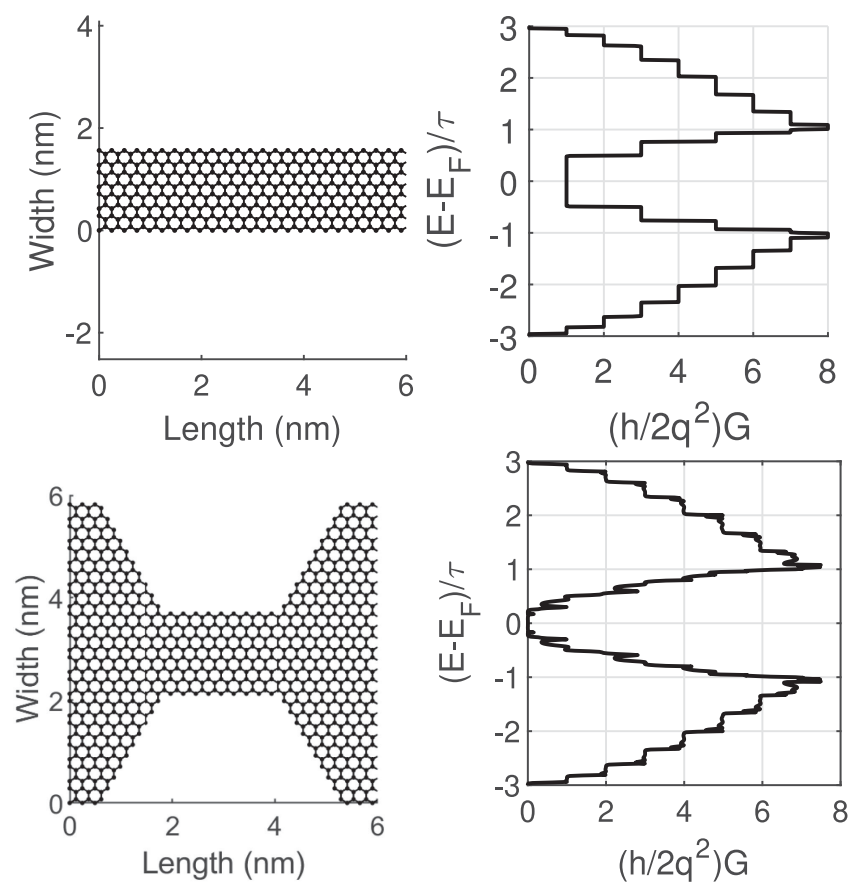

Fig. 1. Top row: The lattice of a graphene nanoribbon and its conductance dispersion diagram shown on the right side of the top row. The absence of band gap is evident. Bottom row: A butterfly shaped GNR, the geometry of which results in a small band gap around the Fermi energy level (zero in the y-axis).

of the conductance of the described GNR structure with the presence of defects. We examine the conductance modification for the following cases: (a) defect distribution in the channel, (b) defect distribution in the contacts, and (c) the concentration of defects in each GNR region.

The TBH is given by:

$$
H=-\tau \sum_{i, j} \hat{c}_{i} \hat{c}_{j}^{\dagger},
$$

where $\hat{c}_{i}, \hat{c}_{j}^{\dagger}$ are the annihilation and creation operators respectively and $\tau$ is the overlap integral that has been computed to be equal to about $-3 \mathrm{eV}$ [34]. In our approach, we are taking into consideration only first nearest neighbour interactions, meaning that we neglect every other interaction between non-direct neighbouring carbon atoms. Increasing the range of atoms' effective neighbourhood significantly increases computational complexity, while offering only insignificant increase to the method's precision.

After the introduction of the GNR geometry through the TBH and electron hopping between atoms, we use the NEGF method to calculate its conductance. Briefly, NEGF method consists of 4 main equations. The $1^{\text {st }}$ step is the computation of the retarded Green's function using Eq. (2):

$$
G^{R}=\left[E I-H-\Sigma_{L}-\Sigma_{R}\right]^{-1},
$$

where $E$ stands for the energy of the electrons transported through the nanoribbon and $I$ is the identity matrix, which shares the same dimensions with the tight-binding Hamiltonian $H$ (Eq. (1)). Even though NEGF can be used for simulating devices with any number of contacts [35], in our case we are utilizing 


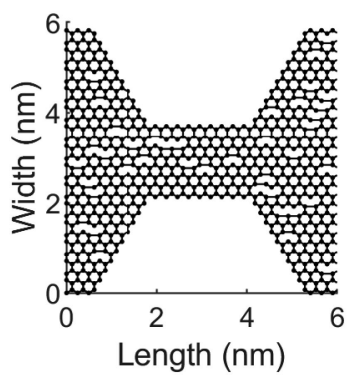

(a)

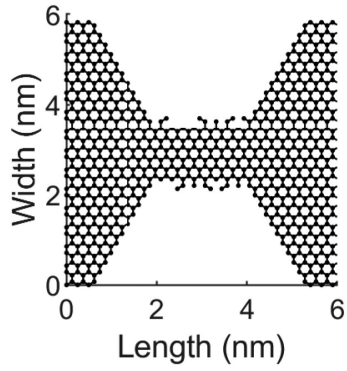

(d)

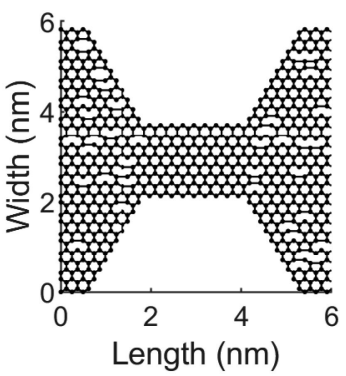

(b)

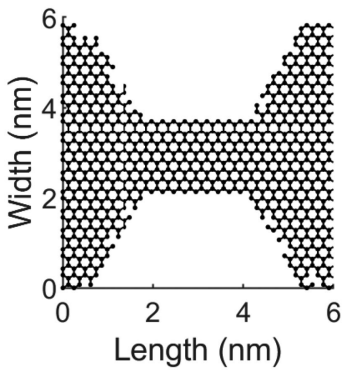

(e)

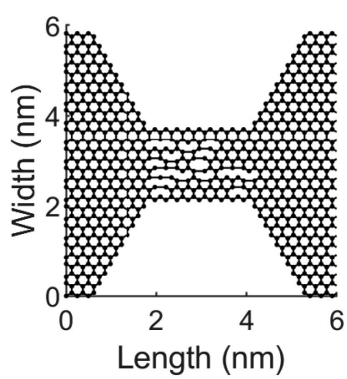

(c)

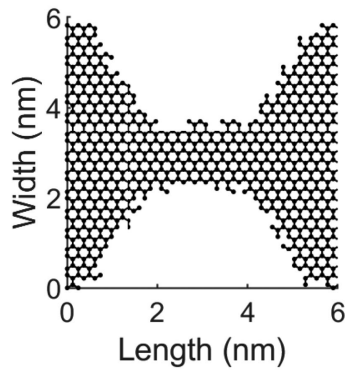

(f)

Fig. 2. Graphene lattice with (a) Bulk defects at channel and contact regions, (b) Bulk defects only at the contact region, (c) Bulk defects only at the channel region, (d) Edge defects only at the channel region, (e) Edge defects only at the contact region, (f) Edge defects at the channel and contact region.

only two contacts. So, $\Sigma_{L}$ and $\Sigma_{R}$ are the self-energies of the left and the right contact respectively. Taking into consideration the above formulations, the advanced Green's function can be computed as: $G^{A}=\left(G^{R}\right)^{\dagger}$.

The $2^{\text {nd }}$ equation of the NEGF method is:

$$
G^{n}=G^{R} \sum^{i n} G^{A},
$$

where $G^{n}$ physically represents the density of electrons at a specific energy level $E$. The term $\Sigma^{\text {in }}$ practically reflects the total power of an external source (i.e. the applied potential difference between the left and right ohmic contact of a device) and is calculated by the following equation:

$$
\Sigma^{i n}=f_{L} \Gamma_{L}+f_{R} \Gamma_{R} .
$$

Here, $f_{L}$ and $f_{R}$ denote the Fermi energy of the left and right contact, respectively. $\Gamma_{L}$ and $\Gamma_{R}$ are the broadening factors of left and right contact respectively, which are calculated as a function of the aforementioned self-energies $\Sigma_{L}$ and $\Sigma_{R}$. The broadening factors physically describe the hoping of electrons from the ohmic contacts to the main conductor.

The $3^{r d}$ equation of the NEGF method derives from Eq. (2) and calculates the density of states (DoS) as follows:

$$
A=i\left(G^{R}-G^{A}\right) .
$$

Finally, the $4^{\text {th }}$ and last equation of the method is used to compute the conductance of the system, as a function of electron energy, between the two contacts:

$$
G(E)=\frac{2 q^{2}}{h} \operatorname{Trace}\left[\Gamma_{L} G^{R} \Gamma_{R} G^{A}\right] .
$$

As mentioned before, GNRs with zigzag edge configuration have been proven to have a semi-metallic behavior instead of a much desired semiconducting one [36]. The zero band-gap problem is therefore a great obstacle in the use of graphene nanoribbons for the realizations of new graphene based switching devices. There is a lot of research going on around the zero band-gap problem, and several solutions have been proposed, like the vertical stacking of graphene layers for the realization of bi- and multi-layer graphene [37] and the growth of graphene on specific substrates [38]. Additionally, the narrowing of one of GNR's dimensions has been proposed as an effective way for realizing band-gap. Recent research has exploited that exact property for the realization of GFET like devices with the use of butterfly shaped GNRs [32].

This geometry of graphene, that enables band gap engineering of GNR based devices, has been expanded with the utilization of devices with different shaped GNRs. The proposed devices when combined together can lead to computing architectures similar to that of CMOS, but with much better performance in terms of delay [23]. This makes such devices a very promising alternative to silicon based structures. However, the reliability, viability and practical exploitation of those, cannot be validated without an extensive analysis on their behavior when under the effect of lattice defects (Fig. 2).

\section{DEFECTS IN GNRS}

In this Section, the types of defects considered in the GNR lattice as shown in Fig. 4(a) are presented. In particular, the single vacancy (SV) in different configurations is shown, and, in more details, the standard single vacancy in Fig. 4(b) and, the case where a bond between two of the three available carbon atoms is formed, the corresponding single vacancy (Fig. 4(c)). These defects are described in the literature as a single vacancy $V 1(5-9)$ 


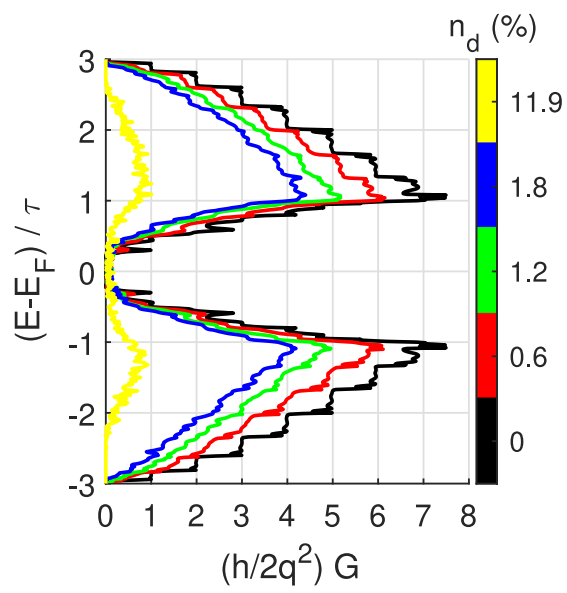

(a)

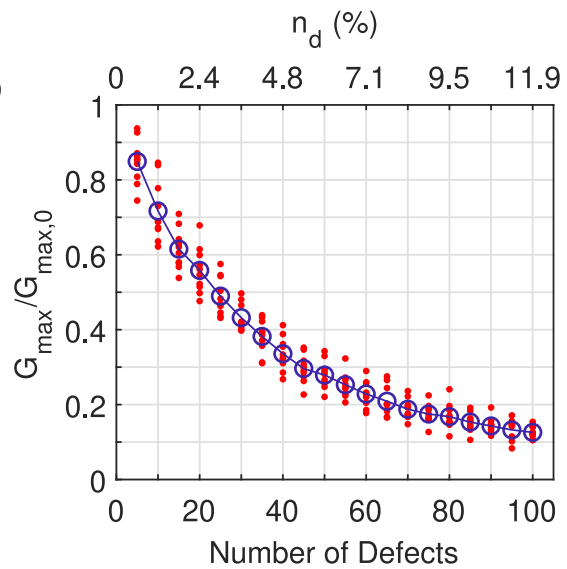

(b)

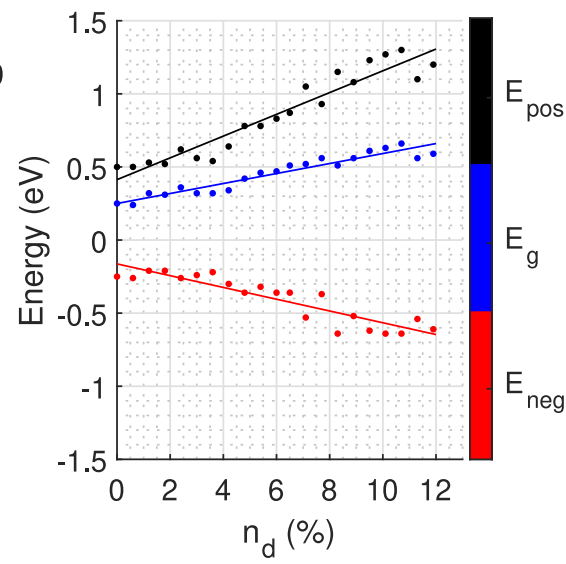

(c)

Fig. 3. (a) Energy dispersion diagrams of the conductance calculated for different number of defects in GNR lattices shown in Fig. 2(a). (b) The dependence of the maximum conductance of a GNR on the number of lattice defects. The average values of $G$ for each $n_{d}$ are varying smoothly (solid line). (c) The change of maximum energy of valence band, minimum energy of conduction band, and energy gap with the increase of bulk defect density on both contact and channel region, while straight lines are the fitting lines for every variable.

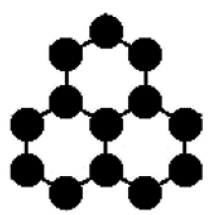

(a)

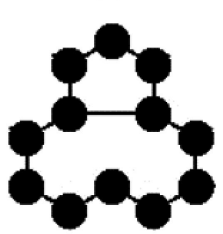

(c)

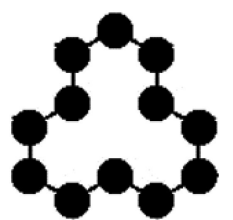

(b)

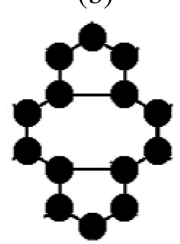

(d)
Fig. 4. Graphene lattice with (a) no defect, (b) a single vacancy (SV), (c) a single vacancy with two available carbon atoms bonded $V 1(5-9)$ and (d) a double vacancy $V 2(5-8-5)$.

due to the appearance of two different grid structures, where one of them (the top defined in the image above) consists of 5 atoms, while the other consists of 9 atoms [3], respectively. The formation and the migration energies of this defect are $7.4 \mathrm{eV}$ and $1.7 \mathrm{eV}$, respectively [39]. While the length of the normal bond between two carbon atoms is about $0.142 \mathrm{~nm}$ long, the new bond that is formed between two carbon atoms after the vacancy, has a length of $r=(0.142 \times \sqrt{(3)}) \mathrm{nm}$. This different bond is introduced in the model through the tight binding overlap parameter. For the original bond of $0.142 \mathrm{~nm}$, the overlap parameter has been experimentally determined to be about $-3 \mathrm{eV}$. The new overlap integral $\tau$ can be calculated by:

$$
\tau_{0}=-3 \times \frac{1.42}{r^{2}}
$$

Moreover, the expansion of this phenomenon can lead to the creation of the so called in literature, double vacancy $V 2(5-8-5)$, as shown in Fig. 4(d). This is the most thermodynamic stable configuration and the migration energy of $\mathrm{V} 2$ is $7 \mathrm{eV}$, denoting that $V 2$ is almost stationary. In addition, in case of $V 1$ there is always a dangling bond while in case of $V 2$ there is no dangling bond, meaning that $V 1$ is chemically reactive while $V 2$ is not.

Our investigations begin by simulating the case where the whole GNR lattice contains defects, except for the edges. The number of defects is varied in the range of 5 to 100 . The calculations were executed in an additive way, meaning that 5 more vacancies were added in a random way to the previously existing formation. This process was repeated (for 10 times) and the final results were averaged. This procedure was followed in all simulations presented in this work, unless otherwise specified. Such typical deffective GNR lattice is shown in Fig. 2(a).

Figure 3(a) presents the effect of defect number on the electronic properties of the GNR utilizing conductance energy dispersion diagrams. The calculated conductance $G$ is expressed in quantum conductance units, $\frac{2 q^{2}}{h}$ where $q$ is the elementary charge and $h$ is the Planck's constant. As previously mentioned, each diagram is an average of 10 different simulations for the same number of defects in the GNR lattice. Considering that the total number of carbon atoms in the simulated GNR are 840 , then the number of defects from 5 to 100 correspond to a concentration $n_{d}$ in the range $0.6-12 \%$. Obviously, the results in Fig. 3(b) reveal that $G$ is lowering as the $n_{d}$ increases. Moreover, the $G(E)$ curves are not symmetric with respect to the Fermi level $E_{F}$ (or the neutrality point) and this asymmetry becomes more evident for energies higher than the energy where maximum conductance $G_{\max }$ is observed. This finding suggests that the GNR conductance is affected in a different manner for electrons and holes. Nevertheless, the energy gap $E_{g}$ around the neutrality point remains constant for all examined values of $n_{d}$. The latter result is mainly attributed to the fact that the bulk disorder in a GNR with perfect edges does not affect the carrier transport taking place mainly from the edges. It should be here emphasized that the energy gap $E_{g}$ is not the same 


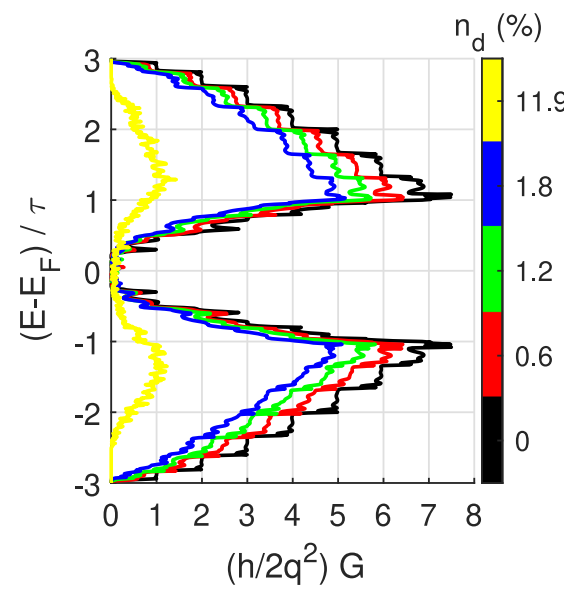

(a)

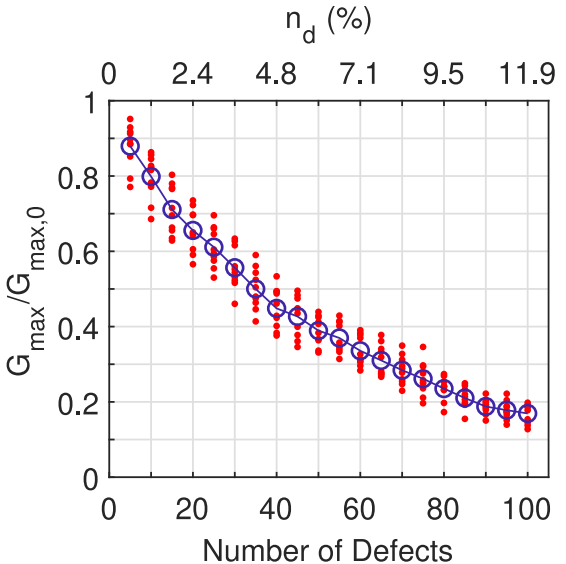

(b)

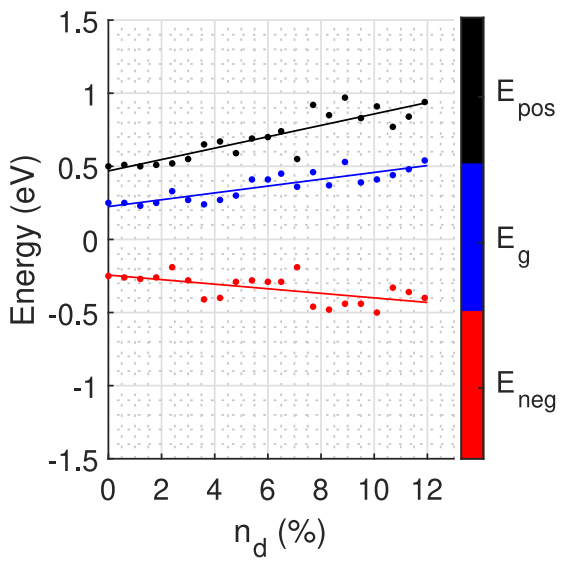

(c)

Fig. 5. (a) Energy dispersion diagrams of the conductance calculated for different numbers of defects in GNR lattices shown in Fig. 2(b). (b) The dependence of the maximum conductance of a GNR on the number of lattice defects on contact region. (c) The change of maximum energy of valence band, minimum energy of conduction band, and energy gap with the increase of bulk defect density on contact region, while straight lines are the fitting lines for every variable.

with that extracted by transport measurements, the transport gap, beyond which the conductivity of the GNR increases very fast, i.e. the change from the OFF state to the ON state. In most cases transport gap is larger due to several GNR device imperfections [40]. Furthermore, the conductance quantization is fading and practically disappears for $n_{d}>1.2 \%$.

In order to investigate further the conductance degradation as a function of the defect number, we examine the variation of $G_{\max }$ at positive energies, e.g. $G_{\max }=6.1$ at $\frac{E-E_{F}}{\tau}=1.1$. For the shake of comparison, $G_{\max }$ is divided with $G_{\max , 0}$ that is the maximum conductance of the perfect GNR (see Fig. 1). Hereafter, the $G_{\max }$ and the normalized $\frac{G_{\max }}{G_{\max , 0}}$ will be used without any difference. In Fig. 3(b), the dependence of $\frac{G_{\max }}{G_{\max , 0}}$ on $n_{d}$ is shown. Evidently, $G_{\max }$ decreases rapidly for $n_{d}<3.6 \%$ and more slowly for higher concentrations.

Another significant parameter that can be potentially of great importance, is the effect of lattice defects on the size of the energy gap $\left(E_{g}\right)$.

For the general case of defects on the whole bulk region of the device, including both the channel and contact regions, Fig. 3(c) shows the change of the $E_{g}$ with the increase of defect concentration on the grid. This figure also presents the change of $E_{\text {pos }}$ and $E_{n e g}$ which correspond to the lowest energy of the conduction band and the highest energy of the valence band, respectively. Those energies are connected with the $E_{g}$, which can be calculated as $E_{g}=\left|E_{\text {pos }}-E_{\text {neg }}\right|$.

Figure 3(c) indicates that $E_{g}$ broadens, while $E_{\text {pos }}$ rises and $E_{n e g}$ decreases. They can be fitted finely on least squares lines whilst those straight lines have different slopes. In particular, the line that describes $E_{\text {pos }}$ has the highest slope, namely $a_{\text {pos }}=0.0744$, the slope of $E_{g}$ follows a value of $a_{g}=0.0342$, while $E_{n e g}$ changes the least with a slope of $a_{n e g}=-0.0403$. It is also obvious that $E_{F}$ does not have an equal distance from $E_{p o s}$ and $E_{n e g}$, but, in this case, it is located closer to $E_{n e g}$. The presented asymmetry is visible on the energy dispersion diagrams of Fig. 3(a). The $E_{g}$ increase can be reasonably explained by the symmetry breaking that creates electron scattering on the graphene surface.

\section{DefeCtS IN THE Wide Regions}

In order to separate the effect of the defects in the contacts from the defects in channel regions, further simulations were performed. More specifically, we constructed lattices where defects were located only in the contact regions and, in particular, only in the bulk region of the GNR, without affecting the edges. Such lattices, used in simulations, are shown in Fig. 2(b).

The conductance dispersion diagrams are shown in Fig. 5(a). In this case, those diagrams indicate a slightly different behavior of the device, in comparison with the previous general case of defects covering both channel and contact regions. Even though the conductance $G$ is always lowering as the $n_{d}$ increases, the ratio of this decrease is smaller. This result is in harmony with the property of GNRs maximum conductance to be affected mainly by the shortest dimension of the grid. Again, the symmetry around $E_{f}$ is not preserved especially for a high number of defects. A very interesting finding is that the effect on the level quantization phenomenon is far inferior in this case. Especially for energies higher than $E_{f}$, conductance quantization is almost perfectly preserved for $n_{d} \leq 1.2 \%$, and also very well preserved for $n_{d}=1.8 \%$, and even higher defect densities.

In Fig. 5(c), the diagram of the $E_{g}$ change provides similar results with the previous case. $E_{\text {pos }}$ and $E_{g}$ are again increasing, while $E_{\text {neg }}$ is decreasing. $E_{\text {pos }}$ increases with a slope of $a_{\text {pos }}=$ $0.4684, E_{n e g}$ decreases with a slope of $a_{n e g}=-0.2437$ and $E_{g}$ is the variable that changes with the lowest slope of the three, $a_{g}=0.2246$, but very close in terms of absolute value to the slope of $E_{\text {pos }}$.

\section{Defects In the Channel Region}

According to the results of the previous section, it is apparent that the defects in the channel should govern the conductance 


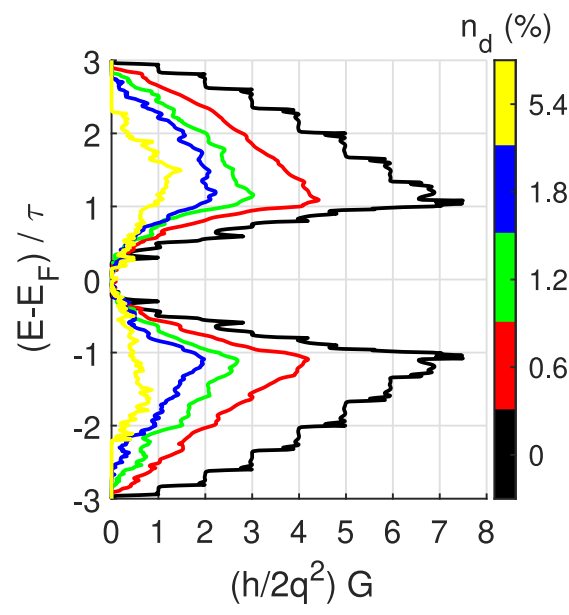

(a)

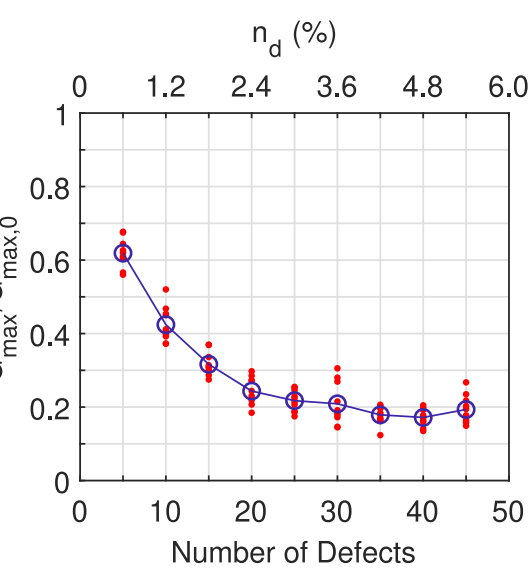

(b)

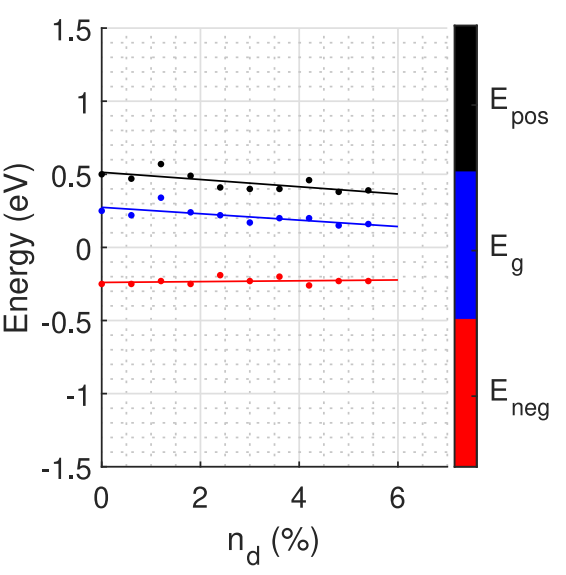

(c)

Fig. 6. (a) Energy dispersion diagrams of the conductance calculated for different number of defects in GNR lattices shown in Fig. 2(c). (b) The dependence of the maximum conductance of a GNR on the number of lattice defects on channel region. (c) The change of maximum energy of valence band, minimum energy of conduction band, and energy gap with the increase of bulk defect density on channel region, while straight lines are the fitting lines for every variable.

lowering in the under study GNR structure. For the purpose of deeper understanding, the effect of defects in GNR lattice only in the channel region was investigated according to Fig. 2(c).

The related energy dispersion of the conductance is shown in Fig. 6(a) and the dependence of the maximum conductance on the increase of the defects is presented in Fig. 6(b). The total number of defects in the channel region that was examined, was up to $5.4 \%$ of the total number of atoms in the device. Such a concentration of defects, restricted in the channel region is destructive for the device. Thus, further increase of the defect densities surely does not give any significant results.

Fig. 6(c) describes the change of $E_{\text {pos }}, E_{g}$ and $E_{n e g}$ with the increase of defect densities at the edges of the device channel regions. For the first time here, an increase on the defect density leads to the decrease of the $E_{p o s}$. This is accompanied by a very small increase of the $E_{n e g}$ and both lead to a decrease of $E_{g}$, meaning practically to a smaller energy gap. In particular, the slope of $E_{p o s}$ decrease is equal to $a_{p o s}=-0.0247$, which is very close the slope of $E_{g}$ decrease, i.e. $a_{g}=-0.219$. The rate by which $E_{n e g}$ increases is very small and equal to $a_{n e g}=0.0028$. This practically means that the line that describes the change of $E_{n e g}$ is almost straight. It is also the reason why the change rates of $E_{\text {pos }}$ and $E_{g}$ are almost the same.

In the following Sections, we present our simulation results regarding the influence of the edge defects in a GNR with no bulk disorder on the conductance.

\section{Defects at THE ChANNEl EdgES}

The channel region has proven to be the most significant one, in terms of affecting the electric properties, and, most important, the conductance of GNR based devices. This phenomenon is attributed to the dominant role of the smallest dimension of a nanoribbon sheet on the device's operation. The same phenomenon applies when dealing with defects concentrations

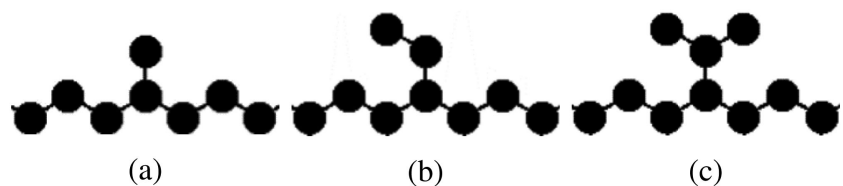

Fig. 7. Graphene lattice with zigzag edge with three different type of defects: (a) single $\mathrm{C}$ atom, (b) two $\mathrm{C}$ atoms and (c) three $\mathrm{C}$ atoms.

only at the edges. The effect of the channel edge defects on the conductance are examined. Typical configurations of edge defects considered in our simulations are demonstrated in Fig. 7. Obviously, the presence of edge defects lead to a local shortening of the channel region width.

Lattices with a range of 1 to 36 defects were tested with the incremental step of the number of vacancies being 1 . The selected step is smaller than the previous one because the number of atoms comprising the edges, is by far smaller. Also, due to the significant effect of the edges on electron transport properties, a more early and abrupt change on the conductance of the device is expected.

In Fig. 2(d), a typical grid with defective channel edges is presented. The extreme case of the two whole rows of atoms missing, one from the top and one from the bottom, is of great interest. This leads to a grid which preserves its symmetry and zig-zag shape, but has a smaller channel width, which is reduced by $(2+\sqrt{2}) \alpha$.

Fig. 8(a) shows the calculated dispersion diagram indicating the effect of defective grids on the device's operation for various numbers of defects. It is clear that after a certain number of defects, the conductance on the device starts to increase again, in accordance with relevant results in the field [41], until the extreme case of 36 defects was considered, where perfect shaped quantised levels reappear. This time the number of levels is 


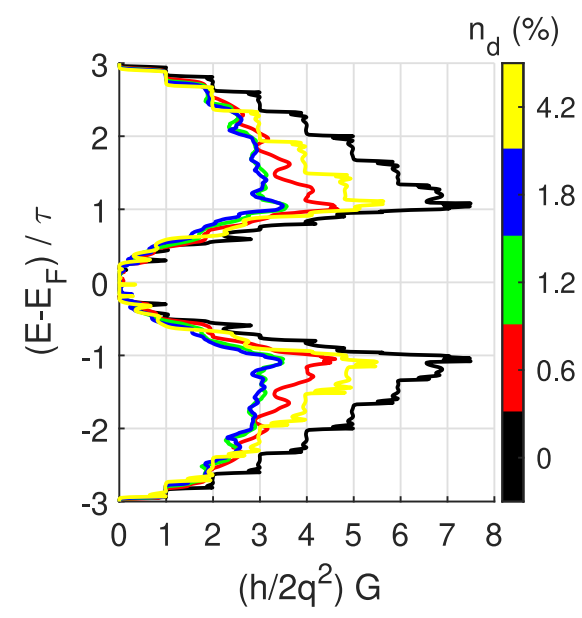

(a)

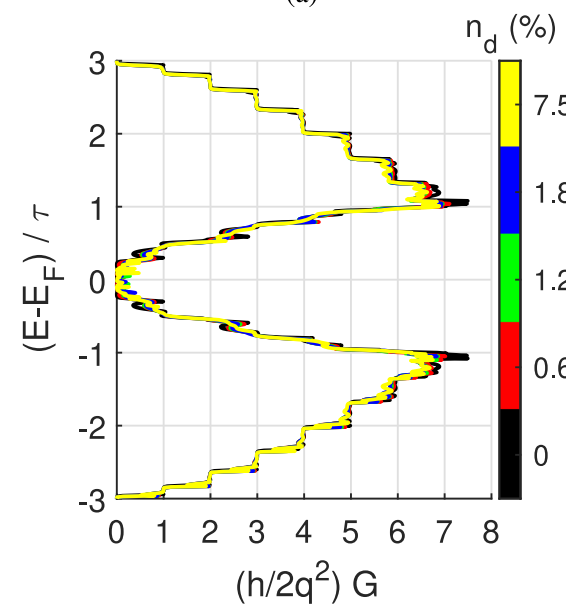

(d)

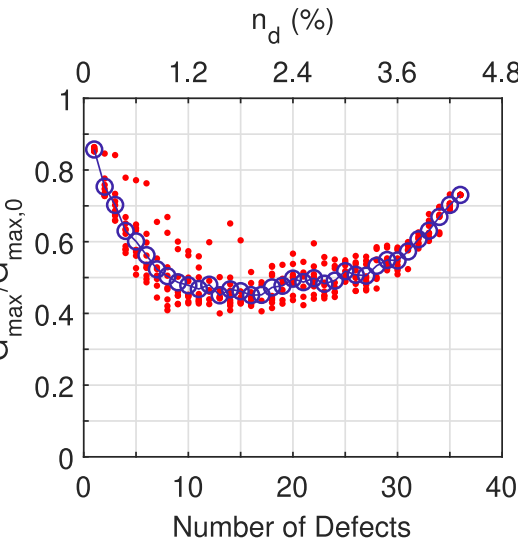

(b)

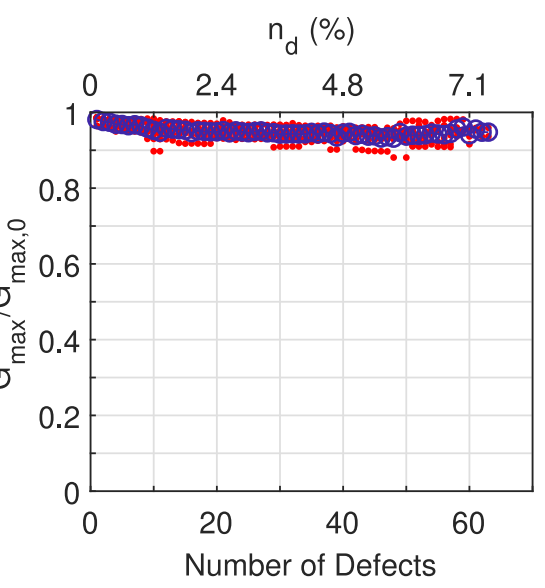

(e)

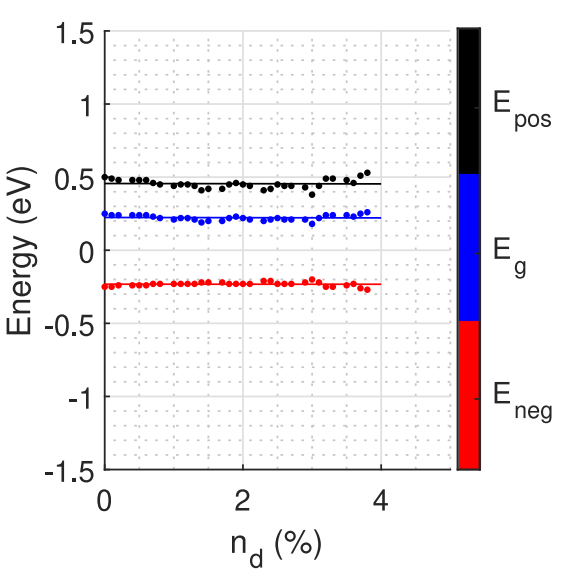

(c)

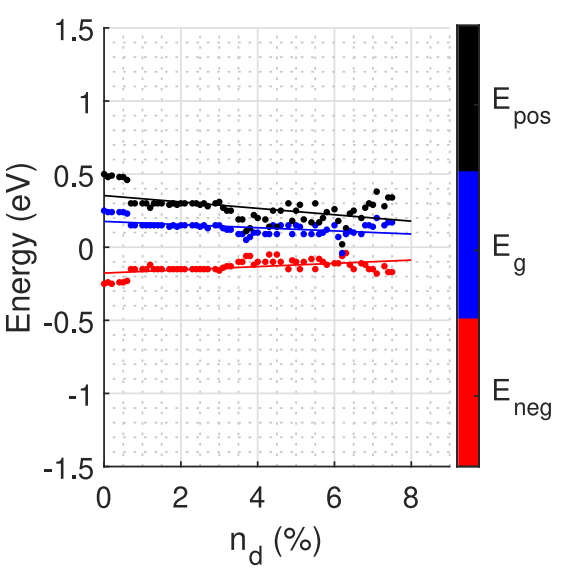

(f)

Fig. 8. (a), (d) Energy dispersion diagrams of the conductance calculated for different number of defects in GNR lattices shown in Fig. 2(d) and Fig. 2(e), respectively. (b), (e) The dependence of the maximum conductance of a GNR on the number of lattice defects for edge defects only at the channel region and contact region, respectively. (c), (f) The change of maximum energy of valence band, minimum energy of conduction band, and energy gap with the increase of edge defect density on channel region and contact region, respectively, while straight lines are the fitting lines for every variable.

smaller, due to the smaller width of the channel region, which directly affects the quantization.

The maximum conductance for the different number of defects, is presented in Fig. 8(b), where the red dots are the values for each iteration, while the blue line and the blue circles represent the mean value of conductance for each number of defects. From the Fig. 8(b), it is obvious that after about 18 edge defects, about half the number of defects resulting to the extreme case previously described, the maximum normalized conductance starts to increase again. So, when the zigzag edges regain their dominance, the performance of the device increases.

Fig. 8(c) presents the changes of $E_{p o s}, E_{g}$ and $E_{n e g}$ with the increase of defect concentration, respectively. In this case, the fitting lines are almost totally straight, and parallel with each other. More specifically, the slope for each line is $a_{p o s}=-0.0007$, $a_{g}=-0.0008, a_{n e g}=-0.0001$ for $E_{p o s}, E_{g}$ and $E_{n e g}$ in accordance. There seems to be very small connection between the energy gap and the number of defects of the channel edges of butterfly shaped GNR.

\section{Defects at The Contacts' EdGeS}

In the following simulations, only the defects on the edges of the wide regions were considered, while no new bonds after each vacancy were formed. In Fig. 2(e), a typical case of a defective GNR lattice with defects at the contacts' edges is presented. Lattices with a range of 1 to 63 defects were tested with the incremental step of the number of vacancies being equal to 1. 10 sets of those simulation were performed. The transition from one step to another, in each set, was made in an additive way, meaning that 1 more vacancy was added to the previously existent formation.

In this case, both dispersion diagrams of Fig 8(d) and maximum conductance diagram of Fig 8(e) lead to the same conclusions. It is obvious that the edges of the contact region of the GNR play an inconsiderable role on affecting its conductance. Fig 8(e) shows that even for relatively high defect densities on the edges, namely for densities up to $n_{d}=7.3 \%$, the dispersion diagrams are only slightly affected, and even the quantization levels remain almost totally unspoiled. Also, Fig 8(d) shows 


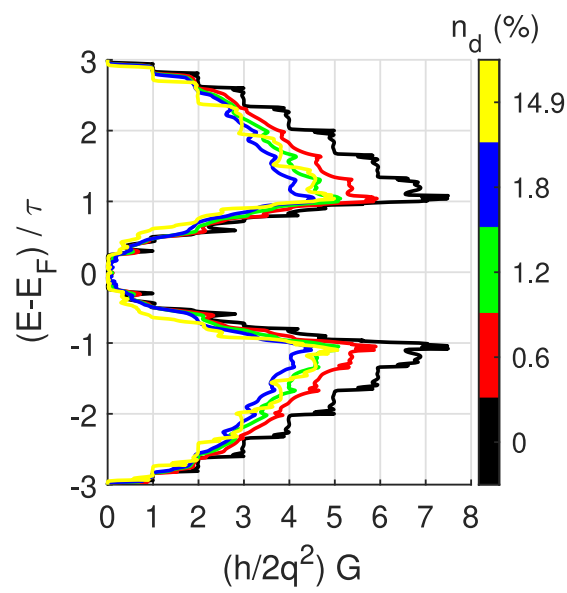

(a)

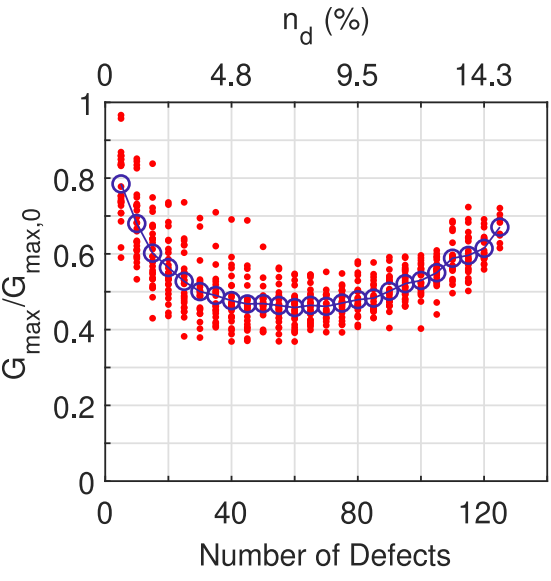

(b)

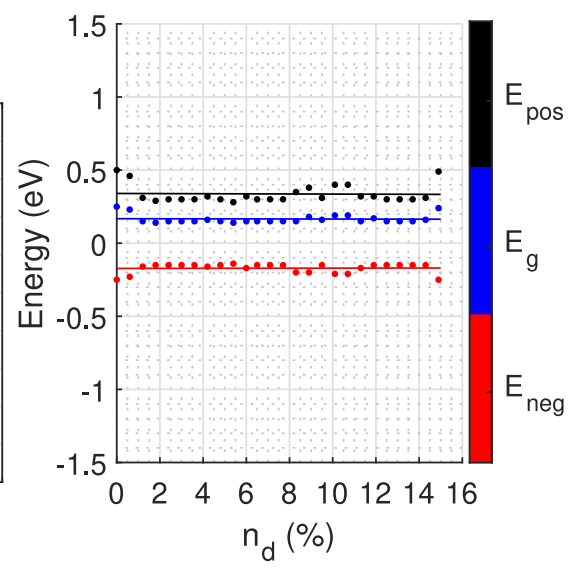

(c)

Fig. 9. (a) Energy dispersion diagrams of the conductance calculated for different number of defects in GNR lattices shown in Fig. 2 f. (b) The dependence of the maximum conductance of a GNR on the number of lattice defects on both channel and contact regions. (c) The change of maximum energy of valence band, minimum energy of conduction band, and energy gap with the increase of edge defect density on both channel and contact regions, while straight lines are the fitting lines for every variable.

with a better resolution that the performance of the device in terms of maximum conductance practically remains intact. The maximum conductance of a defective GNR drops only by $6.81 \%$ in comparison with the ideal GNR.

Fig. $8 \mathrm{f}$ describes the correlation between the values of $E_{\text {pos }}, E_{g}$ and $E_{\text {neg }}$ and the number of defects on edges of the contacts of a butterfly shaped GNR. In this case, there is a strong tendency of the $E_{\text {pos }}$ and $E_{g}$ to decrease. On the other hand, $E_{n e g}$ increases. The fitting lines for $E_{p o s}, E_{g}$ and $E_{n e g}$ change with a slope of $a_{p o s}=-0.0219, a_{g}=-0.0108$ and $a_{n e g}=0.0111$ respectively. Here we can observe that although for defect densities lower than $3 \%$, the fitting lines seem to be in accordance with the change of the values, for $n_{d}>3$ the fitting is not so accurate. However, it is clear, that $E_{g}$ is getting smaller with the increase of defect densities.

\section{Defects AT THE GNR EdGeS}

As a final test case, we simulated devices that have defects on their edges, both on the wide contact and also on the short channel region, in order to examine the effect of edge defects on the device properties as a whole. In Fig. $2 \mathrm{f}$ an instance of the defective grid is presented; it is basically a combination of the grids shown in Fig 2(d) and Fig 2(e).

This time grids with a range of 5 to 125 defects were examined. Each time the number of defects was increased by at least 5 more. In Fig. 9(a), like in the previous cases, you can see the initial energy dispersion diagrams

In Fig. 9(c) the effect of edge defects on both the channel and the contact region together on the values of $E_{p o s}, E_{g}$ and $E_{n e g}$ is tested. Except from a small number of outliers, the values of the energies with the increase of defect densities seem to fit very well on almost straight lines. More precise, the values change with a slope of $a_{p o s}=-0.0004 a_{g}=-0.0002$ and $a_{\text {neg }}=-0.0002$ for $E_{\text {pos }}, E_{g}$ and $E_{\text {neg }}$ respectively. For up to $n_{d}=15 \%$ defective grid, where the defects are located only

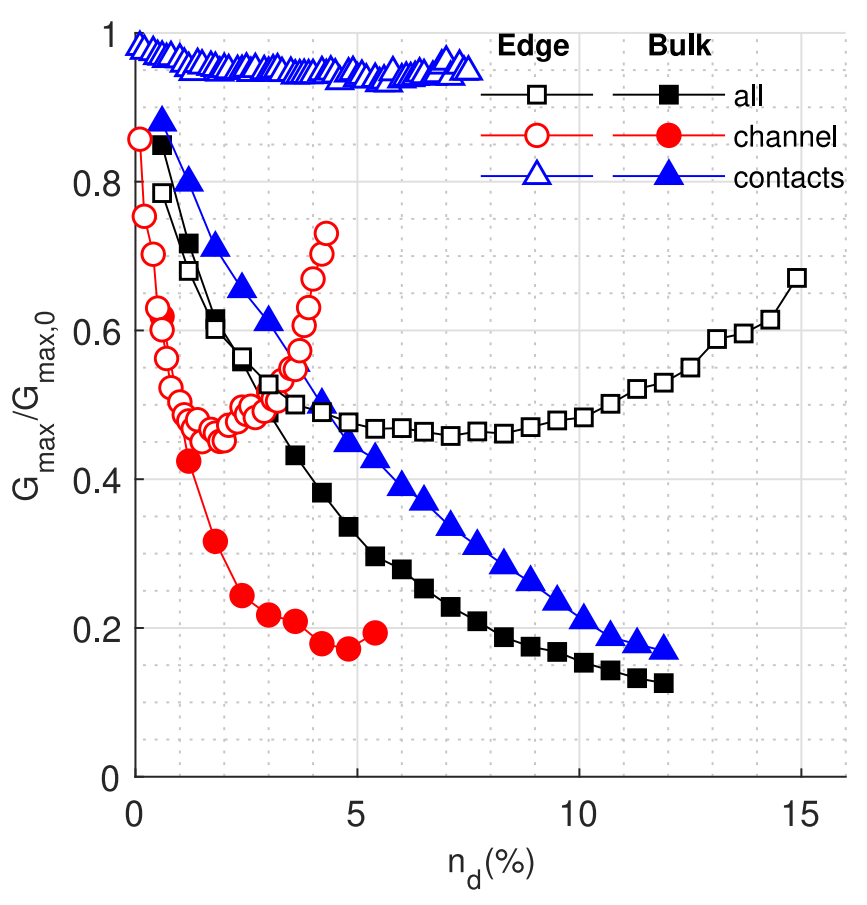

Fig. 10. Overall comparison of the change of conductance with the increase of defect density, for every case described above.

on the edges of a butterfly based device, the energy gap seems to be unaffected.

Fig. 10 is a general comparison of the maximum conductance of a defective device, for every case that has been already presented in this paper. The first observation that can be made is that there is a very similar behavior between the cases of bulk channel defects and the bulk defects on the whole area of the device. This showcases the impact of the contacts as the dominant, which is mainly credited to the higher number of atoms that is included in the contact regions, in comparison with 
the amount of atoms at the channel. The maximum difference between those cases in terms of maximum conductance can be seen for defect concentrations of defects around $n_{d}=5.5 \%$, which is the same value where a device with bulk channel-only defects reaches its minimum value. Even though bulk channel defects lead rapidly the device to a minimum value of maximum conductance, all the cases of bulk defects end up almost to the same value of conductance, which is about $80 \%$ smaller than the initial of the perfect device.

In the cases of edge defects, the results are different. More specific, in the case of edge contact defects, the impact on the devices' maximum conductance is insignificant. This is the only case where defects do not affect significantly the operation of the device. The devices with channel edge defects as well as those combining both contact and channel edge defects develop a behavior that was not present before. The minimum value of maximum conductance is obtained when half of the targeted edge atoms are missing. This value is considerably higher in comparison with the values that occur at the cases of bulk defects, but is achieved for very low defect concentrations, namely about $n_{d}=2-4 \%$. This time the performance is reduced only by $55 \%$. A further increase of the defects leads to an improved performance which reaches up to $68 \%$ and $74 \%$ of the initial performance, for edge defects on both channel and contacts, and only on the channel region, respectively. This maximum value after removing two layers of edge atoms is mainly determined by the new width of the channel region.

\section{CONCLUSION}

In this paper, we investigated the operation of butterfly shaped, GNR based devices, under non-ideal conditions. In particular, we investigated the effect of defects on the electronic properties of the devices. We considered defects on specific parts of the device for each different simulation, in order to find out which part of the device is more defect-tolerant. We also investigated whether different parts of the device affect its properties in different ways. For the simulations presented, we used the very accurate NEGF combined with $\mathrm{TBH}$, which we expanded and added the capability of including different kinds of defects. We tested the most significant variables that define the device switching capabilities, namely the maximum conductance, the energy gap and also the conductance of the device at different energies.

Our results indicate that lattice defects affect significantly the electronic properties of the device's. In general, the channel region appears to be more severely affected by defects than the contact region. Both edge and bulk channel defects reduce the maximum conductance to very low values. The shape and symmetry of the edges play a very significant role in this phenomenon. In the cases where channel edge defects are included, the maximum conductance increases, when perfect zig-zag becomes dominant again. The energy quantization property that is present on an ideal defect-free grid, vanishes for very small defect concentrations, in almost every sub-location of the device, except from the edge defects on the contacts. Another significant variable, the energy gap, changes with the increase of defects.
Bulk defects at the contacts induce an increase of device energy gap, while edge and channel defects either decrease the gap, creating problems on device's operation, or do not affect it at all.

\section{ACKNOWLEDGMENT}

The authors would like to thank Vasileios Ntinas for general feedback and help with data visualisation.

\section{REFERENCES}

[1] K. S. Novoselov et al., "Electric field effect in atomically thin carbon films," Science, vol. 306, no. 5696, pp. 666-669, 2004.

[2] K. S. Novoselov et al., "Room-temperature quantum hall effect in graphene," Science, vol. 315, no. 5817, pp. 1379-1379, 2007.

[3] F. Banhart, J. Kotakoski, and A. V. Krasheninnikov, "Structural defects in graphene," ACS Nano, vol. 5, no. 1, pp. 26-41, 2011.

[4] H.-T. Chin, J.-J. Lee, M. Hofmann, and Y.-P. Hsieh, "Impact of growth rate on graphene lattice-defect formation within a single crystalline domain," Sci. Rep., vol. 8, no. 1, pp. 1-6, Dec. 2018.

[5] Y. Zhang et al., "Invisible growth of microstructural defects in graphene chemical vapor deposition on copper foil," Carbon, vol. 96, pp. 237-242, 2016.

[6] L. Liu, M. Qing, Y. Wang, and S. Chen, "Defects in graphene: Generation, healing, and their effects on the properties of graphene: A review," J. Mater Sci. Technol., vol. 31, no. 6, pp. 599-606, 2015.

[7] W. Tian, W. Li, W. Yu, and X. Liu, "A review on lattice defects in graphene: Types, generation, effects and regulation," Micromachines, vol. 8, no. 5, p. 163, May 2017.

[8] L. G. Cançado et al., "Quantifying defects in graphene via raman spectroscopy at different excitation energies," Nano Lett., vol. 11, no. 8, pp. 3190-3196, 2011.

[9] J. C. Meyer, C. Kisielowski, R. Erni, M. D. Rossell, M. F. Crommie, and A. Zettl, "Direct imaging of lattice atoms and topological defects in graphene membranes," Nano Lett., vol. 8, no. 11, pp. 3582-3586, 2008

[10] A. Lherbier, S. M.-M. Dubois, X. Declerck, Y.-M. Niquet, S. Roche, and J.-C. Charlier, "Transport properties of graphene containing structural defects," Phys. Rev. B, vol. 86, Aug. 2012, Art. no. 075402.

[11] W. Zhang, W.-C. Lu, H.-X. Zhang, K. M. Ho, and C. Z. Wang, "Tightbinding calculation studies of vacancy and adatom defects in graphene," J. Phys.: Condens. Matter, vol. 28, no. 11, Feb. 2016, Art. no. 115001.

[12] S. H. M. Jafri et al., "Conductivity engineering of graphene by defect formation," J. Phys. D: Appl. Phys., vol. 43, no. 4, Jan. 2010, Art. no. 045404.

[13] S. Song, T. Kim, O. Sul, W. Shin, and B. Cho, "Improvement of graphenemetal contact resistance by introducing edge contacts at graphene under metal," Appl. Phys. Lett., vol. 104, May 2014, Art. no. 183506.

[14] Y. Na, J. Han, and J.-S. Yeo, "Approaches to reduce the contact resistance by the formation of covalent contacts in graphene thin film transistors," Appl. Sci. Convergence Technol., vol. 26, pp. 55-61, Jul. 2017.

[15] Y. Chen, A. Sangai, M. Gholipour, and D. Chen, "Graphene nano-ribbon field-effect transistors as future low-power devices," in Proc. Int. Symp. Low Power Electron. Des., 2013, pp. 151-156.

[16] N. Harada et al., "Graphene-gate transistors for gas sensing and threshold control," in Proc. IEEE Int. Electron Devices Meeting. 2016, pp. 18.2.1-18.2.4

[17] X. Li, X. Wang, L. Zhang, S. Lee, and H. Dai, "Chemically derived, ultrasmooth graphene nanoribbon semiconductors," Science, vol. 319, no. 5867, pp. $1229-1232,2008$.

[18] C. Backes et al., "Production and processing of graphene and related materials," 2D Mater, vol. 7, no. 2, Jan. 2020, Art. no. 022001.

[19] T. C. Li and S.-P. Lu, "Quantum conductance of graphene nanoribbons with edge defects," Phys. Rev. B, vol. 77, Feb. 2008, Art. no. 085408.

[20] E. Mucciolo, A. Castro Neto, and C. Lewenkopf, "Conductance quantization and transport gap in disordered graphene nanoribbons," Phys. Rev. B, vol. 79, Feb. 2009, Art. no. 075407.

[21] K. Rallis, P. Dimitrakis, G. C. Sirakoulis, I. Karafyllidis, and A. Rubio, "Effect of lattice defects on the transport properties of graphene nanoribbon," in Proc. IEEE/ACM Int. Symp. Nanoscale Architectures, 2019, pp. 1-2.

[22] I. Nikiforidis, I. G. Karafyllidis, and P. Dimitrakis, "Simulation and parametric analysis of graphene p-n junctions with two rectangular top gates and a single back gate," J. Phys. D: Appl. Phys., vol. 51, no. 7, Jan. 2018, Art. no. 075303. 
[23] Y. Jiang, N. C. Laurenciu, H. Wang, and S. D. Cotofana, "Graphene nanoribbon based complementary logic gates and circuits," IEEE Trans. Nanotechnol., vol. 18, pp. 287-298, 2019.

[24] Y. Jiang, N. C. Laurenciu, and S. D. Cotofana, "On carving basic Boolean functions on graphene nanoribbons conduction maps," in IEEE Int. Symp. Circuits Syst., 2018, pp. 1-5.

[25] K. Rallis, G. C. Sirakoulis, I. Karafyllidis, and A. Rubio, "Multi-valued logic circuits on graphene quantum point contact devices," in Proc. IEEE/ACM Int. Symp. Nanoscale Architectures, 2018, pp. 1-5.

[26] S. Moysidis, I. G. Karafyllidis, and P. Dimitrakis, "Graphene logic gates," IEEE Trans. Nanotechnol., vol. 17, no. 4, pp. 852-859, Jul. 2018.

[27] S. Moysidis, I. G. Karafyllidis, and P. Dimitrakis, "Design and simulation of graphene majority gate without back-gating," Eng. Res. Exp., vol. 1, no. 1 , Sep. 2019, Art. no. 015030.

[28] C. Bena and G. Montambaux, "Remarks on the tight-binding model of graphene," New J. Phys., vol. 11, no. 9, 2009, Art. no. 095003.

[29] S. Reich, J. Maultzsch, C. Thomsen, and P. Ordejon, "Tight-binding description of graphene," Phys. Rev. B, vol. 66, no. 3, 2002, Art. no. 035412

[30] S. Datta, Lessons From Nanoelectronics: A New Perspective on Transport. Singapore: World Scientific Publishing Co. Pte. Ltd., 2012.

[31] S. Datta, "Nanoscale device modeling: The green's function method," Superlattices Microstruct., vol. 28, no. 4, pp. 253-278, 2000.

[32] I. G. Karafyllidis, "Current switching in graphene quantum point contacts," IEEE Trans. Nanotechnol., vol. 13, no. 4, pp. 820-824, Jul. 2014.

[33] Y. Lee et al., "Tunable valley splitting due to topological orbital magnetic moment in bilayer graphene quantum point contacts," Phys. Rev. Lett., vol. 124, no. 12, 2020, Art. no. 126802

[34] L. Chico, L. X. Benedict, S. G. Louie, and M. L. Cohen, "Quantum conductance of carbon nanotubes with defects," Phys. Rev. B, vol. 54, pp. 2600-2606, Jul. 1996.

[35] S. Moysidis and I. G. Karafyllidis, "Conductance of 1-shaped and t-shaped graphene nanoribbons," Microelectron. J., vol. 72, pp. 11-13, 2018.

[36] K. Nakada, M. Fujita, G. Dresselhaus, and M. S. Dresselhaus, "Edge state in graphene ribbons: Nanometer size effect and edge shape dependence," Phys. Rev. B, vol. 54, no. 24, 1996, Art no. 17954.

[37] A. H. Castro Neto, F. Guinea, N. M. R. Peres, K. S. Novoselov, and A. K. Geim, "The electronic properties of graphene," Rev. Mod. Phys., vol. 81, pp. 109-162, Jan. 2009.

[38] S. Zhou et al., "Substrate-induced bandgap opening in epitaxial graphene," Nat. Mater., vol. 6, pp. 770-775, 2007.

[39] X. Li et al., "Graphene and related two-dimensional materials: Structureproperty relationships for electronics and optoelectronics," Appl. Phys. Rev., vol. 4, no. 2, 2017, Art. no. 021306.

[40] R. Murali, Graphene Nanoelectronics: From Materials to Circuits. New York, NY, USA: Springer, 2012.

[41] M. Poljak, E. B. Song, M. Wang, T. Suligoj, and K. L. Wang, "Influence of edge defects, vacancies, and potential fluctuations on transport properties of extremely scaled graphene nanoribbons," IEEE Trans. Electron Devices, vol. 59, no. 12, pp. 3231-3238, Dec. 2012.

Konstantinos Rallis (Member, IEEE) received the Diploma Eng. in electrical and computer engineering from the Democritus University of Thrace, Komotini, Greece, in 2018 and he is currently working toward the Ph.D. degree. His research interests include nanoelectronic devices and circuits.
Panagiotis Dimitrakis (Senior Member, IEEE) received the B.S. and M.S. degrees in physics from the University of Athens, Athens, Greece, in 1995 and 1998, respectively, and the Ph.D. degree from the National Technical University of Athens, Athens, Greece, in 2006. He has the authored more than 60 papers in peer review journals in the field of semiconductor devices, nonvolatile memories and organic electronics. He is the Editor of the book series Charge-trapping nonvolatile memories and has organized various international conferences worldwide and edited their proceedings. In 2007, he joined NCSR Demokritos, where he is currently a Senior Researcher of the Institute of Nanoscience and Nanotechnology and the Manager of the central Nanotechnology and Microsystems Cleanroom Facility.

Ioannis Karafyllidis received the Diploma Eng. and Ph.D. degrees in electrical engineering from the Aristotle University of Thessaloniki, Thessaloniki, Greece. In 1992, he joined as a Faculty Member with the Department of Electrical and Computer Engineering, Democritus University of Thrace, Komotini, Greece, where he is currently a Professor. His current research interests include quantum computing, modeling and simulation of nanoelectronic devices and circuits, and biological networks modeling. He is a Fellow of the Institute of Nanotechnology, a Founding Member of the American Academy of Nanomedicine and a Member of the Technical Chamber of Greece.

Antonio Rubio (Senior Member, IEEE) received the M.Sc. and Ph.D. degrees from the Industrial Engineering Faculty, Polytechnic University of Catalonia (UPC), Barcelona, Spain. He has been an Associate Professor with the Electronic Engineering Department, UPC, and a Professor with the Physics Department, Balearic Islands University, Palma, Spain. He is currently a Professor of electronic technology with the Telecommunication Engineering Faculty, UPC. His research interests include VLSI design and test, device and circuit modeling, high-speed circuit design, and new emerging nanodevices and nanoarchitectures. $\mathrm{He}$ is an Associate Editor for the IEEE TRANSACTIONS ON COMPUTERS, a Senior Editor of the IEEE TRANSACTIONS OF NANOTECHNOLOGY, the Chair Elect of Nano-Giga TC, and the IEEE Computer Sciences Integrity Chair

Georgios Ch. Sirakoulis (Member, IEEE) received the Diploma Eng. and $\mathrm{Ph} . \mathrm{D}$. degrees in electrical and computer engineering from the Democritus University of Thrace (DUTh), Komotini, Greece, in 1996 and 2001, respectively. $\mathrm{He}$ is currently a Professor with the Department of Electrical and Computer Engineering, DUTh. He has authored or coauthored more than 250 technical papers, he is a co-editor of seven books, coauthor of 25 book chapters and Guest Editor in 14 Special Issues. His current research interests include complex electronic systems, future and emergent electronic devices, circuits, models and architectures, unconventional computing memristors, cellular automata, quantum cellular automata, bioinspired computation or biocomputation and bioengineering and modeling and simulation. $\mathrm{He}$ is an Associate Editor of the IEEE TRANSACTIONS OF NANOTECHNOLOGY, the IEEE TRANSACTIONS ON COMPUTERS, the IEEE TRANSACTIONS ON CIRCUITS AND SYSTEMS, Microelectronics Journal, Integration, the VLSI Journal, and Journal of Cellular Automata. 